\title{
A Comparison of the Relative Safety and Efficacy of Laparoscopic Choledochotomy with Primary Closure and Endoscopic Treatment for Bile Duct Stones in Patients with Cholelithiasis
}

\author{
Shuang Liu, MS, ${ }^{1}$ Changzhong Fang, MS, ${ }^{2}$ JingWang Tan, MD, ${ }^{3}$ and Wenliang Chen, $\mathrm{MS}^{4}$
}

\begin{abstract}
Background: To date, several clinical trials have demonstated that both one-stage laparoscopic cholecystectomy (LC) combined with common bile duct exploration (LC+BDE) with primary closure and one-stage LC combined with endoscopic stone extraction (LC+ESE) are the two primary clinical approaches to treat cholelithiasis. However, no studies to date have directly compared the LC+BDE with primary closure and onestage LC+ESE procedures. We, therefore, conducted a retrospective analysis of patients with cholelithiasis who had been treated through LC+ESE or LC+BDE to compare these two approaches for the treatment of cholecystitis and common bile duct stones (CCBDS).

Methods: Consecutive CCBDS patients with cholelithiasis in our hospital who were diagnosed through Media Resource Control Protocol (MRCP) and ultrasound between June 2010 and February 2017 were randomly assigned to undergo either LC+ESE or LC+BDE, as both procedures are routinely used to treat cholelithiasis in our hospital. All patients were made aware of the risks and benefits of the surgery preoperatively, and this study was approved by the ethics committee of our institute. Outcomes in these two groups, including rates of success and reasons for operative failure, were then compared, as were data pertaining to patient demographics, clinical findings, postoperative stay duration, and medical expenses. In addition, biliary reflux as measured through computed tomography or gastrointestinal imaging was monitored for a minimum of 2 years.

Results: In total, 207 CCBDS patients were identified during the study period and were randomized into the $\mathrm{LC}+\mathrm{ESE}(n=103)$ or LC+BDE $(n=104)$ treatment groups. We found that patients treated through LC+BDE achieved a significantly higher success rate than that achieved in patients treated through LC+ESE (93.3\% versus $82.5 \% ; P<.05)$. Specifically, the $\mathrm{LC}+\mathrm{BDE}$ with primary closure procedure failed in patients with impacted stones located at the end of the common bile duct (CBD) and in those with stenosis of the sphincter of Oddi. The only variable that differed significantly between these two treatment groups was stone location. Variables other than stone location, CBD size, and stone size did not differ significantly between the two groups. However, the LC+BDE treatment was associated with significant reductions in patient operating time, morbidity, hospital day duration, and biliary reflux of duodenal contents relative to the LC+ESE treatment.

Conclusions: We found that LC+BDE with primary closure was a safer and more effective means of treated CCBDS patients than was the LC+ESE procedure and that it was not associated with risks of sphincterotomy of duodenal papilla (EST)- or T-tube-related complications. However, our data also clearly indicate that LC+BDE cannot replace LC+ESE in all patients, and that as such both approaches should be considered as being complementary to one another, with their relative advantages in a given patient being defined based upon local resource availability and expertise. In addition, when the LC+ESE procedure fails then the LC+BDE treatment can be safely employed as a salvage approach.
\end{abstract}

\footnotetext{
${ }^{1}$ Graduate Department of Shanxi Medical University, Taiyuan, China.

${ }^{2}$ Department of General Surgery, The Second Hospital of Shanxi Medical University, Taiyuan, China.

${ }^{3}$ Department of General Surgery, Chinese PLA General Hospital, Beijing, China.

${ }^{4}$ Department of General Surgery, The Second Affiliated Hospital of Shanxi Medical University, Taiyuan, China.
} 
Keywords: bile duct exploration, endoscopic stone extraction, cholelithiasis, laparoscopic cholecystectomy, primary closure

\section{Background}

$\mathbf{R}$ OUGHLY $10 \%-18 \%$ of patients affected by cholelithiasis who have common bile duct (CBD) stones are treated through cholecystectomy. ${ }^{1,2}$ However, optimal treatment and patient management strategies in individuals with cholelithiasis accompanied by cholecystitis and common bile duct stones (CCBDS) remain controversial. The most prevalent treatment strategies for CBD stones are either open cholecystectomy and $\mathrm{CBD}$ exploration (BDE) or laparoscopic cholecystectomy (LC) with either BDE or endoscopic stone extraction (ESE), which can be performed either pre- or postcholecystectomy in two stages.

This approach is most often combined with sphincterotomy or sphincteroplasty to achieve CBD clearance. ${ }^{3-6}$ The relative strengths and weaknesses of these different approaches have been assessed in numerous clinical trials to date. Different institutions typically favor either a one-stage $\mathrm{LC}+\mathrm{ESE}$ approach or an LC+BDE with primary closure approach, but no studies to date have been published comparing the relative benefits of these two strategies. ${ }^{5,7}$ As such, in the present prospective randomized trial, we compared operative outcomes in CCBDS patients with cholelithiasis treated through these two approaches.

\section{Methods}

\section{Patients}

Patients enrolled in this study were consecutive patients from our hospital who had presented with symptomatic gallstones or CCBDS between June 2010 and February 2017. Patients were initially diagnosed based upon elevated liver function tests, jaundice, cholangitis, biliary pancreatitis, or imaging findings. Patients were excluded from this study if they had undergone retrograde cholangiopancreatography (ERC) before their referral for cholecystectomy or if they had severe pancreatitis or cholangitis that necessitated immediate endoscopic retrograde cholangiopancreatography (ERCP drainage). After hospital admission and subsequent ultrasound (US) and Media Resource Control Protocol (MRCP) analyses to confirm the presence of bile duct stones or CBD dilation, patients who provided informed consent to participate in this study received conservative treatments, including standard prophylactic antibiotics and anisodamine (654-2).

In cases of small CBD stones $(<6 \mathrm{~mm})$, patients were administered for $5 \mathrm{mg}$ dexamethasone for at least 3 days. In patients exhibiting marked reductions in jaundice or other symptoms consistent with the suspected elimination of the stone from the CBD, additional MRCP analyses were performed to determine whether the stone had indeed migrated out of the CBD. When no stones were visible in the CBD upon MRCP reanalysis, patients were treated through LC alone. All other patients were assigned to one of two treatment groups: the LC+BDE group (assigned to undergo LC and BDE with primary closure), and the LC+ESE group (assigned to undergo one-stage LC and calculi extraction through ERCP) (Fig. 1 Patients were randomized to undergo either LC+ESE or LC+BDE by the intended method). Operative success was defined based on the successful completion of all surgical operations, including the complete clearance of CBD and cholecystectomy.

Potential causes of operative failure included failed surgical procedures, residual stone detection, or the need to convert the LC procedure to an open surgical procedure. Outcome rates were then compared between these two groups. The design of this study was consistent with the Declaration of Helsinki as subsequently modified by the Chinese Health and Medical Research Council on Human Experiments and on Scientific Practice.

\section{Operative outcomes}

Laparoscopic cholecystectomy+bile duct exploration. All patients received prophylactic antibiotics before surgery. Laparoscopic exposure was achieved with a telescoping $11 \mathrm{~mm}$ trocar and two 5-mm subcostal ports. Intraoperative examination served to confirm the presence of

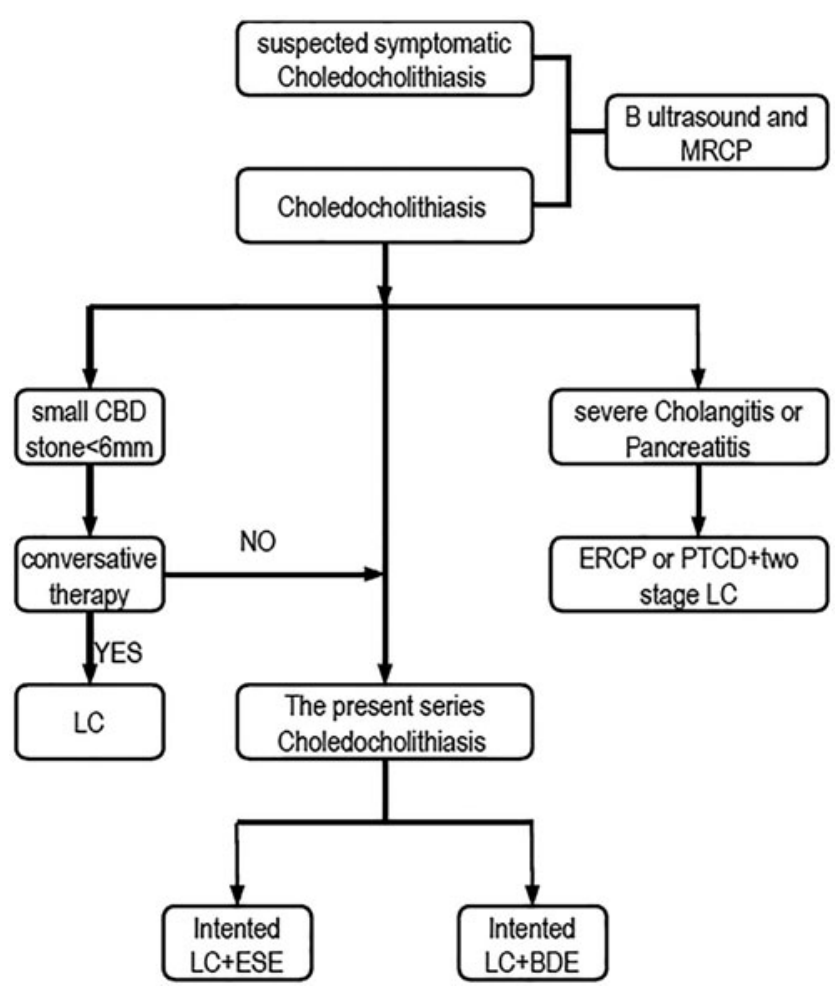

FIG. 1. Patients were randomized to either LC+ESE or $\mathrm{LC}+\mathrm{BDE}$ by intended method. BDE, bile duct exploration; $\mathrm{CBD}$, common bile duct calculi; ERCP, endoscopic retrograde cholangiopancreatography; ESE, endoscopic stone extraction; LC, laparoscopic cholecystectomy; MRCP, Media Resource Control Protocol; PTCD, percutaneous biliary drainage radiography. 
suspected CBD stones, which were then removed through choledochotomy-mediated clearance. In brief, supraduodenal exposure of the CBD was performed and an incision that was sufficiently long to permit easy stone delivery was made, after which stones were cleared through irrigation, Dormia basket deployment with flexible choledochoscopic guidance, and Fogarty balloon sweeps. Choledochoscopy was then used to confirm stone removal, with small CBD $(<0.8 \mathrm{~mm})$ having been indicated in the series. The incision was then closed using monofilament absorbable sutures. Drain decompression of the cleared bile duct was performed in patients with evidence of stenosis or edematous thickening of the ampulla as a consequence of either the operation or as a result of local inflammation and fibrosis.

Laparoscopic cholecystectomy+endoscopic stone extraction. Patients in the LC+ESE group underwent ERCP bile duct clearance through one-stage cholecystectomy. Side viewing duodenoscopes were used, and needle knife papillotomes were employed in cases when precut papillotomy was required. Mechanical and extracorporeal shock wave lithotripsy was available as needed. Sphincter balloon dilation was not used in these patients as an alternative to sphincterotomy for the purposes of stone removal. The operative duration was defined as the time between the initiation of surgery and the completion of ERCP procedures. Hospital stay duration was defined as the time from the date of surgery until discharge. Bile leak was defined based on the presence of bile within the subhepatic peritoneal suction drains. Trial endpoints included operative time, morbidity, rates of stone retention, rates of reoperation, and hospital stay duration. ${ }^{8}$

Participating surgeons were experienced laparoscopic biliary surgeons with extensive documented experienced carrying out laparoscopic choledochotomy and suture techniques.

\section{Follow-up and recurrence}

After patient discharge from the hospital, follow-up assessments of liver function and US imaging were conducted every 3-6 months, or in any case where patients exhibited symptoms consistent with possible recurrence. The presence of biliary duodenal reflux and stone recurrence was also confirmed through US, computed tomography (CT) imaging, cholangiography, and/or gastrointestinal (GI) imaging in patients as appropriate. Biliary duodenal reflux was defined as the detection of biliary air in US or CT images or the detection of the reflux of duodenal contents into the biliary duct in GI or CT images. Recurrence was defined as the development of cholangitis or of recurrent stones.

\section{Statistical analysis}

Means were calculated for all continuous variables and were used for comparisons between groups. Data were compared through Mann-Whitney $U$-tests and chi-squared tests, as appropriate, with SPSS v13.0 used for all statistical testing. Risk factors associated with recurrence were assessed through logistic regression analyses. $P<.05$ was the significance threshold.

\section{Results}

\section{Patient demographics}

For the study period from June 2010 to February 2017, a total of 351 patients were admitted to our hospital with elevated liver function test results, jaundice, biliary pancreatitis, cholangitis, and $\mathrm{CBD}$ stones that were detected through MRCP. Of these patients, 20 required immediate treatment due to severe cholangitis or pancreatitis and were excluded from this study, whereas an additional 79 patients were excluded due to having undergone ESE before admission, and 45 patients had stones $<6 \mathrm{~mm}$ in size that were cleared successfully through conservative treatment. The remaining 207 patients were randomly assigned to undergo either LC+ESE or LC+BDE ( $n=103$ and 104, respectively). There were no significant demographic differences between these patient groups (Table 1).

\section{Rates of operative success}

In a total of 18 patients in the $\mathrm{LC}+\mathrm{ESE}$ group, CBD stone removal was not successful. Reasons for failure included difficulties in stone retrieval due to large stone size $(>1.5 \mathrm{~cm}$; 5 patients), an inability to cannulate the Ampulla of Vater ( 2 patients), the presence of residual stones ( 5 patients), or the need to repeat ESE or to convert to open surgery (6 patients). In contrast, CBD stone removal failed in only 7 of the 104

Table 1. Demographics of Patient With Intended LAPAROSCOPIC CHOLECYSTECTOMY+ENDOSCOPIC STONE EXTRACTION AND LAPAROSCOPIC Cholecystectomy+Bile Duct Exploration

\begin{tabular}{|c|c|c|c|}
\hline & $\begin{array}{c}L C+E S E \\
\mathrm{n}=103\end{array}$ & $\begin{array}{c}L C+B D E \\
\mathrm{n}=104\end{array}$ & $\mathrm{P}$ \\
\hline Age (years) mean (range) & $57.1(18-81)$ & $56.9(19-78)$ & $>0.5$ \\
\hline Male/female & $42 / 61$ & $41 / 63$ & $>0.5$ \\
\hline ASA $1-5$ status (median) & $2(1-3)$ & $2(1-3)$ & $>0.5$ \\
\hline Comorbidities & 18 & 21 & $>0.5$ \\
\hline Hypertension & 7 & 8 & \\
\hline Diabetes mellitus & 2 & 3 & \\
\hline Ischemic heart disease & 3 & 4 & \\
\hline Renal failure & 1 & 0 & \\
\hline COPD & 2 & 2 & \\
\hline Presenting complaints & 47 & 56 & $>0.5$ \\
\hline Abdominal pain & 47 & 56 & \\
\hline Jaundice & 42 & 45 & \\
\hline Cholangitis & 42 & 45 & \\
\hline Pancreatitis & 4 & 5 & \\
\hline $\begin{array}{l}\text { Serum bilirubin, } \\
\text { mol/L (mean) }\end{array}$ & $47.5 \pm 11.7$ & $45.1 \pm 13.4$ & $>0.5$ \\
\hline $\begin{array}{l}\text { White cell count, } \\
\text { 109/L (mean) }\end{array}$ & $11.8 \pm 2.7$ & $12.0 \pm 3.4$ & $>0.5$ \\
\hline Stone size of CBD & $1.2 \pm 0.3$ & $1.1 \pm 0.4$ & $>0.5$ \\
\hline Diameter of CBD & $1.3 \pm 0.3$ & $1.2 \pm 0.4$ & $>0.5$ \\
\hline Impacted stone & 4 & 3 & $>0.5$ \\
\hline Stone $>1.5 \mathrm{~cm}$ & 5 & 5 & $>0.5$ \\
\hline SO stenosis & 2 & 2 & $>0.5$ \\
\hline
\end{tabular}

BDE, bile duct exploration; CBD, common bile duct; COPD, chronic obstructive pulmonary disease; ESE, endoscopic stone extraction; LC, laparoscopic cholecystectomy; SO, sphincter of Oddi. 
Table 2. The Successful Rate and Causes of Unresolved Choledocholithiasis by Intended Method

\begin{tabular}{|c|c|c|c|}
\hline & $L C+E S E, \mathrm{~N}=103$ & $L C+B D E, \mathrm{~N}=104$ & $\mathrm{P}$ \\
\hline & $82.5 \%$ & $93.3 \%$ & $<0$ \\
\hline \multirow[t]{4}{*}{ Failure cause $(n)$} & Residual stone (not specified) (5) & Residual stone (not specified) (1) & \\
\hline & Stone $>1.5 \mathrm{~cm}(5)$ & Impacted stone at lower end of CBD (3) & \\
\hline & Impossibility in cannulating the Ampulla of Vater (2) & T-tube drainage due to SO stenosis (2) & \\
\hline & Conversion to open (6) & Conversion to open (1) & \\
\hline Convert to opposite & 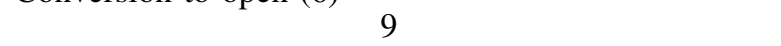 & 3 & \\
\hline
\end{tabular}

BDE, bile duct exploration; CBD, common bile duct; ESE, endoscopic stone extraction; LC, laparoscopic cholecystectomy; SO, sphincter of Oddi.

patients assigned to the LC+BDE group, with reasons for failure, including the presence of impacted stones at the lower end of the CBD (3 patients), the presence of residual stones (1 patient), T-tube drainage due to sphincter of Oddi (SO) stenosis ( 2 patients), and conversion to open surgery (1 patient) (Table 2). In 9 of the patients in whom the LC+ESE approach failed, conversion to one-stage LC+BDE with primary closure was successful. Similarly, in 3 patients in whom the LC+BDE approach failed, conversion to one- or two-stage ESE was successful. The causes of operative failure are summarized in Table 2.

\section{Comparison of operative outcomes between patients treated through ESE and BDE}

Patient operative outcomes are summarized in Table 3 . The average operating time and blood loss levels were significantly greater in the LC+ESE group relative to the LC+BDE group. During postoperative recovery, common complications included pancreatitis, bleeding, and cholangitis in the LC+ESE group. Of the 21 patients with postoperative pancreatitis, the disease was mild-to-moderate in all cases based on Glasgow scores, and only 2 of these patients required admission to the ICU. Four patients in this treatment group suffered mild postoperative bleeding that resolved with observation in a few days. In the $\mathrm{LC}+\mathrm{BDE}$ group, only 2 patients suffered from

Table 3. The Operative Outcome in Patient WITH INTENDED ENDOSCOPIC STONE EXTRACTION AND LBDE GROUP

\begin{tabular}{lccc}
\hline & $\begin{array}{c}\text { ESE group, } \\
\mathrm{n}=103\end{array}$ & $\begin{array}{c}\text { BDE group, } \\
\mathrm{n}=104\end{array}$ & $\mathrm{P}$ \\
\hline Operating time & $171 \pm 46$ & $151 \pm 38$ & $<0.05$ \\
Estimated loss of & $384 \pm 210$ & $179 \pm 83$ & $<0.05$ \\
$\quad$ bleeding & 30 & 18 & $<0.05$ \\
Morbidity (\%) & 4 & 0 & \\
$\quad$ Bleeding & 21 & 2 & \\
Pancreatitis & 5 & 0 & \\
Cholangitis & 0 & 2 & \\
Bile leak & 11 & 16 & \\
Other & $6(2-91)$ & $4(2-9)$ & $<0.05$ \\
Postoperative hospital & & & \\
$\quad$ stay (days) & $29 \pm 4.1$ & $18 \pm 2.3$ & $<0.05$ \\
Cost ( $\times 1000)$ & & & \\
\hline
\end{tabular}

BDE, bile duct exploration; ESE, endoscopic stone extraction; LBDE, laparoscopic bile duct exploration. postoperative pancreatitis and 2 suffered from bile leakage. In both cases, patient symptoms resolved with observation in a few days. These data thus indicated that patients treated through the LC+ESE approach had significantly higher costs, morbidity rates, and hospital stay duration than did patients treated through the LC+BDE approach.

\section{Long-term outcomes in patients successfully treated through ESE and BDE}

Patients were followed for an average of 45 months (range: 12-76), over which time 29 patients exhibited biliary duodenal reflux, including 27 in the successful LC+ESE group and 2 in the successful LC+BDE group. Recurrence of CBD stones was observed in 8 of 85 patients (9.4\%) in the successful LC+ESE group, which was a significantly higher rate than that what was observed in the successful LC+BDE group $(2 / 97 ; 2.1 \%)$. Recurrence of cholangitis was observed in $7 / 85$ patients in the successful LC+ESE group (8.2\%), and in 3/97 patients in the successful LC+BDE group (3.1\%) (Table 4). No patients exhibited any evidence of biliary injury or stricture at any point during the follow-up period.

\section{Variables associated with the recurrence of $C B D$ stones and cholangitis}

Univariate analyses revealed that there was no significant relationship between patient age, gender, comorbidities, preoperative endoscopic sphincterotomy, choledochotomy closure method, or CBD size and the risk of CBD stone recurrence. However, there was a significant association between cholangitis history, treatment method, and CBD stone/cholangitis recurrence (Table 5).

Table 4. The Long-Term Results in Patient with Successful Endoscopic Stone Extraction AND Bile Duct Exploration Group

\begin{tabular}{lccc}
\hline & $\begin{array}{c}\text { ESE group, } \\
\mathrm{N}=85\end{array}$ & $\begin{array}{c}\text { BDE group, } \\
\mathrm{N}=97\end{array}$ & $\mathrm{P}$ \\
\hline Biliary reflux (\%) & 27 & 2 & $<0.05$ \\
Stone recurrence & 8 & 2 & $<0.05$ \\
$\begin{array}{l}\text { Cholangitis } \\
\text { recurrence }\end{array}$ & 7 & 3 & $<0.05$ \\
\hline
\end{tabular}

BDE, bile duct exploration; ESE, endoscopic stone extraction. 
Table 5. Univariate Analysis of FaCtors Associated With COMMON Bile Duct Stone/Cholangitis ReCURRENCE

\begin{tabular}{lccc}
\hline & $\begin{array}{c}\text { Recurrence } \\
(\mathrm{n}=19)\end{array}$ & $\begin{array}{c}\text { No recurrence } \\
(\mathrm{n}=163)\end{array}$ & $\mathrm{P}$ \\
\hline Age (years) & $58.4 \pm 10.1$ & $56.0 \pm 12.3$ & $>0.5$ \\
$\begin{array}{l}\text { Gender } \\
\text { Male }\end{array}$ & 10 & 70 & $>0.5$ \\
$\quad$ Female & 9 & 93 & \\
History of cholangitis & & \\
$\quad$ Yes & 13 & 65 & $<0.01$ \\
$\quad$ No & 6 & 98 & \\
Comorbidity & & & \\
$\quad$ Yes & 3 & 30 & $>0.5$ \\
$\quad$ No & 16 & 136 & \\
Treatment method & & & \\
$\quad$ LC+ESE & 13 & 74 & $>0.01$ \\
LC+BDE & 6 & 89 & \\
Stone size of CBD & & & \\
$>1.5 \mathrm{~cm}$ & 2 & 155 & \\
$\quad<1.5 \mathrm{~cm}$ & 17 & 8.5 & \\
\hline
\end{tabular}

BDE, bile duct exploration; CBD, common bile duct; ESE, endoscopic stone extraction.

\section{Discussion}

CBD stones are a relatively common condition, affecting up to $20 \%$ of people according to some studies. In 10\%-18\% of patients, gallbladder stones located within CBD are often asymptomatic in many patients. ${ }^{9,10}$ The detection and treatment of CBD stones have changed significantly in recent decades, with imaging techniques such as endoscopic US (EUS) and magnetic resonance cholangiography (MRC) now being available to facilitate the visualization of these stones, and endoscopic and laparoscopic techniques being available to treat them. ${ }^{11-15}$ Despite the existence of these tools, the best approaches to diagnosing and removing CBD stones in a costeffective manner remain to be firmly established. The widespread adoption of laparoscopic surgical techniques has led to significant changes in the accuracy, cost, and invasiveness of CBD treatment efforts.

In some patients, optimal management can be based upon ERCP, or on bile duct clearance through laparoscopic choledochotomy or through the cystic duct. For those with suspected stones, precholecystectomy detection with ERCP clearance is frequently employed and has high rates of successful stone clearance. However, between $20 \%$ and $60 \%$ of patients are ultimately found not to have any stones, and in rare cases, serious adverse events can occur in these patients. ${ }^{2,3,9,12}$ Many treatment centers have thus increasingly utilized MRCP and endoscopic US rather than ERCP in an effort to reduce the need for patients to undergo excessive invasive examinations unless they have a significant risk of having CBD stones. ${ }^{11,12,16}$ In patients with a low risk of having CBD stones, LC can generally be conducted without the need for any further assessment, whereas intermediate- to high-risk patients must typically undergo either intraoperative cholangiography (IOC) in a laparoscopy-first approach or ERCP in an endoscopy-first approach. ${ }^{16,17}$
The efficacy of IOC, EUS, and MRC for the diagnosis of CBD stones is reported to be similar. In this study, we assigned low-risk patients to undergo LC directly, whereas intermediate- and high-risk patients were subjected to MRC to detect CBD stones in a dynamic manner to monitor for stone elimination from the bile duct while patients were undergoing conservative therapy, as such migration often occurs in patients with small $(<6 \mathrm{~mm})$ stones. Indeed, MRC is generally considered to be the most accurate noninvasive means of detecting CBD stones, with sensitivity and specificity values of $95 \%$ and $97 \%$, respectively, according to a recent meta-analysis of 67 published controlled trials. ${ }^{16,17}$ In this study, 33/65 (50.7\%) patients with small stones and mild jaundice had stones that had disappeared upon subsequent MRC analysis after 5-7 days of conservative treatment.

Over the course of a median 5-year follow-up period (range: 2-8 years), none of these 33 patients experienced stone recurrence, thus confirming the accuracy of this MRC approach. Importantly, as this MRC is noninvasive and does not expose patients to X-rays, it is ideal for diagnosing CBD stones when available.

At present, there is no strong consensus regarding the optimal treatment for CBD stones. However, recent studies $^{2,7,18}$ have shown that open surgery is superior to LC+ESE or LC+BDE with respect to stone retention and the need for subsequent operations. However, such open surgeries are more invasive, whereas laparoscopic approaches are more complex, time consuming, and require dedicated instruments. As a result, expertise and resource availability is often a more significant driver of the selection of a given CBD stone treatment approach than is cost-effectiveness. The ESE approach has become increasingly popular in recent years in China, but it has multiple disadvantages, including short-term risks of bleeding, pancreatitis, cholangitis, and perforation as well as long-term risks of SO stenosis, reflux of duodenal contents into the $\mathrm{CBD}$, ascending cholangitis, and potentially cholangiocarcinoma. ${ }^{8,19,20}$ One long-term follow-up study found that only $42 \%$ of patients treated through this approach were completely asymptomatic during the follow-up period, whereas $20 \%$ had symptoms of mild cholangitis, and $9 \%$ and $3 \%$ of these patients required one and two additional rounds of ERC, respectively. ${ }^{8}$ Patients treated through this approach have also been shown to exhibit an increased long-term risk of cholangiocarcinoma associated with cholangitis. ${ }^{20} \mathrm{In}$ contrast, the $\mathrm{LC}+\mathrm{BDE}$ approach can allow for clearance of CBD stones without the high risk of morbidity associated with the ESE or open surgical procedures. In most cases, laparoscopic choledochotomy can achieve CBD stone clearance without the need for anterograde sphincterotomy, ${ }^{21}$ thus preserving normal sphincter anatomy and functionality.

Three reviews ${ }^{22-24}$ have shown that the LC+BDE approach is safe and effective as a low-cost means of clearing stones in a single hospitalization with low rates of associated morbidity and mortality. Moreover, we conducted these laparoscopic procedures with primary closure, as have researchers in previous studies. ${ }^{25,26}$ These analyses have revealed that primary closure through bile excision or the transcystic route offers additional benefits of avoiding potential complications associated with T-tube drainage or ESE, with significant reductions in operation time, morbidity, postoperative hospital day duration, and cost without any 
significant increases in rates of stenosis or duodenobiliary reflux relative to T-tube drainage in both open and laparoscopic procedures or LC+ESE. However, the transcystic route depends upon cystic duct patency, the stone, and the compliance of the cystic duct with the necessary cannulation maneuvers, with rates ranging from $73 \%$ to $95 \%$. Success rates associated with this procedure are low, and it requires additional specialized equipment.

As such, the LC+BDE approach may have value as a goldstandard approach to the first-line treatment of patients with CBD stones. With respect to treatment efficacy, in this study we found that stone clearance rates for patients with $\mathrm{CBD}$ stones treated through $\mathrm{LC}+\mathrm{BDE}$ with primary closure were significantly higher than those for patients treated through LC+ESE. In both of these one-stage procedures, similar causes of operative failure were observed, including the need to convert to open surgery as a consequence of severe inflammation or due to laparoscopic failure. In addition, LC+ ESE often failed due to stones being large $(>1.5 \mathrm{~cm})$, an inability to conduct cannulation of the ampulla of Vater, duodenal diverticulum, stone retrieval difficulties, or the presence of multiple stones.

Fortunately, patients with such presentations can generally be treated through $\mathrm{LC}+\mathrm{BDE}$. Even so, $\mathrm{LC}+\mathrm{BDE}$ cannot replace LC+ESE in all patients, and LC+BDE is not amenable to use in patients with impacted stones at the end of the CBD or in patients with a small CBD. In such patients, ESE is instead indicated. As such, LC+BDE and LC+ESE offer complementary therapeutic and clinical value.

\section{Conclusion}

Generally speaking, ERC and ESE remain the preferred strategies for CBD stone detection and clearance in the majority of cases. However, our results suggest that MRC can be used in place of IOC or ERC in a safe and effective manner, making it feasible for use in patients with intermediate- or high-risk choledocholithiasis. Similarly, our results suggest that one-stage LC+BDE with primary closure is at least as feasible as is one-stage LC+ESE in these patients, with the former procedure also offering the advantages of reduced operating time, decreased hospital stay duration, and lower rates of morbidity.

We have, therefore, proposed an approach that can be used to diagnose and treat patients with intermediate- or high-risk choledocholithiasis. This strategy employs the routine use of MRC for CBDS detection, with LC+BDE used as a first-line treatment approach except in cases where the impacted stone is located near the end of the CBD, in patients affected by SO stenosis, or in those in whom the CBD is small $(<7 \mathrm{~mm})$. In patient affected by these issues, ESE may remain a viable complementary approach to stone clearance. Given the potential risk of SO destruction during the ESE procedure, it is essential that all efforts be made to preserve normal SO functionality during stone clearance.

\section{Disclosure Statement}

No competing financial interests exist.

\section{Funding Information}

No funding was received.

\section{References}

1. Ko CW, Lee SP. Epidemiology and natural history of common bile duct stones and prediction of disease. Gastrointest Endosc 2002;56(6 Suppl):S165-S169.

2. Dasari BV, Tan CJ, Gurusamy KS, et al. Surgical versus endoscopic treatment of bile duct stones. Cochrane Database Syst Rev 2013;CD003327.

3. Clayton ES, Connor S, Alexakis N, Leandros E. Metaanalysis of endoscopy and surgery versus surgery alone for common bile duct stones with the gallbladder in situ. Br J Surg 2006;93:1185-1191.

4. Zhu QD, Tao CL, Zhou MT, Yu ZP, Shi HQ, Zhang QY. Primary closure versus T-tube drainage after common bile duct exploration forcholedocholithiasis. Langenbecks Arch Surg 2011;396:53-62.

5. Rogers SJ, Cello JP, Horn JK, et al. Prospective randomized trial of LC+LCBDE vs ERCP/S+LC for common bile duct stone disease. Arch Surg 2010;145:28-33.

6. Rhodes M, Sussman L, Cohen L, Lewis MP. Randomised trial of laparoscopic exploration of common bile duct versus postoperative endoscopic retrograde cholangiography for common bile duct stones. Lancet 1998;351: 159-161.

7. Maple JT, Ikenberry SO, Anderson MA, et al. The role of endoscopy in the management of choledocholithiasis. Gastrointest Endosc 2011;74:731-744.

8. Chen CM, Tay KH, Hoe MN, Salleh I, Lim SH. Endoscopic retrograde cholangiopancreatography management of common bile ductstones in a surgical unit. ANZ J Surg 2005;75:1070-1072.

9. Aerts R, Penninckx F. The burden of gallstone disease in Europe. Aliment Pharmacol Ther 2003;18 Suppl 3:49-53.

10. Menezes N, Marson LP, debeaux AC, Muir IM, Auld CD. Prospective analysis of a scoring system to predict choledocholithiasis. Br J Surg 2000;87:1176-1181.

11. Lacaine F, Corlette MB, Bismuth H. Preoperative evaluation of the risk of common bile duct stones. Arch Surg 1980;115:1114-1116.

12. Wilson TG, Hall JC, Watts JM. Is operative cholangiography always necessary. Br J Surg 1986;73:637-640.

13. Verma D, Kapadia A, Eisen GM, Adler DG. EUS vs MRCP for detection of choledocholithiasis. Gastrointest Endosc 2006;64:248-254.

14. Ainsworth AP, Rafaelsen SR, Wamberg PA, Durup J, Pless TK, Mortensen MB. Is there a difference in diagnostic accuracy and clinical impact betweenendoscopic ultrasonography and magnetic resonance cholangiopancreatography. Endoscopy 2003;35:1029-1032.

15. Romagnuolo J, Bardou M, Rahme E, Joseph L, Reinhold C, Barkun AN. Magnetic resonance cholangiopancreatography: A meta-analysis of test performance in suspected biliary disease. Ann Intern Med 2003;139:547-557.

16. Tse F, Barkun JS, Barkun AN. The elective evaluation of patients with suspected choledocholithiasis undergoing laparoscopic cholecystectomy. Gastrointest Endosc 2004; 60:437-448.

17. Deprez P. Approach of suspected common bile duct stones: Endoscopic ultrasonography. Acta Gastroenterol Belg 2000;63:295-298.

18. Williams EJ, Green J, Beckingham I, Parks R, Martin D, Lombard M. Guidelines on the management of common bile duct stones (CBDS). Gut 2008;57:1004-1021.

19. Cotton PB, Lehman G, Vennes J, et al. Endoscopic sphincterotomy complications and their management: An 
attempt atconsensus. Gastrointest Endosc 1991;37:383393.

20. Tocchi A, Mazzoni G, Liotta G, Lepre L, Cassini D, Miccini M. Late development of bile duct cancer in patients who had biliary-enteric drainagefor benign disease: A follow-up study of more than 1,000 patients. Ann Surg 2001;234:210-214.

21. Macadam RC, Goodall RJ. Long-term symptoms following endoscopic sphincterotomy for common bile ductstones. Surg Endosc 2004;18:363-366.

22. Krauss H, Kern E. Some current problems of biliary tract surgery: Indications and technique ofcholedochotomy, intraoperative cholangiomanometry, primary closure of the common bile duct. Surgery 1967;62:983-987.

23. Martin IJ, Bailey IS, Rhodes M, O'Rourke N, Nathanson L, Fielding G. Towards T-tube free laparoscopic bile duct exploration: A methodologic evolution during 300 consecutive procedures. Ann Surg 1998;228:29-34.

24. Memon MA, Hassaballa H, Memon MI. Laparoscopic common bile duct exploration: The past, the present, and the future. Am J Surg. 2000;179:309-315.
25. Croce E, Golia M, Azzola M, et al. Laparoscopic choledochotomy with primary closure. Follow-up (5-44 months) of 31 patients. Surg Endosc 1996;10:1064-1068.

26. Gurusamy KS, Koti R, Davidson BR. T-tube drainage versus primary closure after laparoscopic common bile ductexploration. Cochrane Database Syst Rev 2013; CD005641.

Address correspondence to: Wenliang Chen, MS Department of General Surgery The Second Affiliated Hospital of Shanxi Medical University No. 382, Wuyi Road Taiyuan 030001 China

E-mail:190693713@qq.com 